\title{
Requirements for Power Hardware-in-the-Loop Emulation of Distribution Grid Challenges
}

\author{
Sebastian Hubschneider \\ Institute of Electric Energy Systems \\ and High-Voltage Technology \\ Karlsruhe, Germany \\ Email: hubschneider@kit.edu
} Karlsruhe Institute of Technology Karlsruhe Institute of Technology Ka

Ingo Mauser Institute of Applied Informatics Institute of Electric Energy Systems and Formal Description Methods Karlsruhe Institute of Technology Karlsruhe, Germany

Email: mauser@kit.edu

\author{
Sebastian Kochanneck \\ Institute of Applied Informatics \\ and Formal Description Methods \\ Karlsruhe, Germany \\ Email: kochanneck@kit.edu
}

Thomas Leibfried and High-Voltage Technology

Karlsruhe Institute of Technology Karlsruhe, Germany

Email: thomas.leibfried@kit.edu

\author{
Bernd Bohnet \\ Institute of \\ Electrical Engineering \\ Karlsruhe Institute of Technolog \\ Karlsruhe, Germany \\ Email: bernd.bohnet@kit.edu
}

Hartmut Schmeck

Institute of Applied Informatics

and Formal Description Methods

Karlsruhe, Germany

Email: schmeck@kit.edu

\author{
Michael Suriyah \\ Institute of Electric Energy Systems \\ and High-Voltage Technology \\ Karlsruhe Institute of Technology \\ Karlsruhe, Germany \\ Email: michael.suriyah@kit.edu
}

Michael Braun
Institute of
Electrical Engineering
sruhe Institute of Technology
Karlsruhe, Germany
ail: michael.braun@ @it.edu

\begin{abstract}
The ongoing transition of low voltage (LV) power grids towards active systems requires novel evaluation and testing concepts, in particular for realistic testing of devices. Power Hardware-in-the-Loop (PHIL) evaluations are a promising approach for this purpose. This paper presents preliminary investigations addressing the systematic design of PHIL applications and their applicable stability mechanisms and gives a detailed review of the related work. A requirement analysis for emulation of grid situations demanding system services is given and the realization of a PHIL setup is demonstrated in a residential scenario, comprising a hybrid electrical energy storage system (HESS).

Index Terms-Hardware-in-the loop simulation, Power distribution faults, Power system simulation, Power quality
\end{abstract}

\section{INTRODUCTION}

The increasing penetration of LV grids with active systems challenges its equipment to deal with negative effects, like overvoltages, harmonics, and transients. These active systems have to contribute actively to system services or have to at least maintain their connection to the grid during a fault and its clearance, i. e. fault ride through (FRT). For testing these capabilities and also the interoperability of active systems according to existing and future grid codes and standards, PHIL promises to be a well suited approach for hardwaredriven evaluations of these properties.

Due to stability challenges caused by inherent system properties, PHIL setups require a careful consideration of the interconnected hard- and software systems. In order to enable these PHIL studies with high accuracy and temporal resolution, the requirements for PHIL scenarios have to be classified regarding feedback and interdependencies.

The paper is structured as follows: Firstly, a short introduction to general PHIL system setup is given followed by a detailed review of PHIL applications in power systems. Then, an in-depth analysis and classification of the requirements for PHIL in LV systems is given. Lastly, results of testing a HESS in a PHIL setup under simulated fault conditions are reported.

\section{POWER HARDWARE-IN-THE-LOOP}

\section{A. Basic System Setup}

Power-Hardware-in-the-Loop (PHIL) extends the approach of Hardware-in-the-Loop, where control signals are exchanged between a device under test (DUT) and a real-time simulator (RTS), by exchanging not only control signals but large scale power signals. This is facilitated by a Power Interface comprising a 4-Quadrant Amplifier for emulating an artificial mains and Measurement Instrumentation for establishing feedback circuits. For coupling the digital real-time simulator with the analog device under test, digital-to-analog- (D/A-) and analog-to-digital-converters (A/D-converters) are needed to close a digital feedback loop. (see Fig. 1)

\section{B. Stability Issues}

As outlined, PHIL setups promise good results in testing real hardware equipment for power applications. However, due to dead-times in the real-time-simulator, the inevitable discretization, calculation times, D/A- and A/D-converter time constants, and time delays caused by the power interface, PHIL struggles with both stability and accuracy issues. Research has been done in order to counteract those issues by designing adequate feedback mechanisms, whereby varying drawbacks in either stability or accuracy have to be accepted (see Section III-E). The basic ideas to improve stability are:

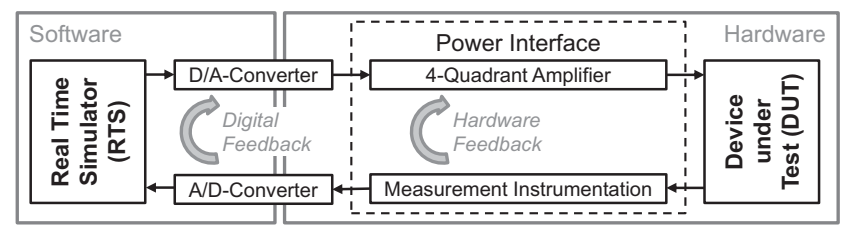

Fig. 1. Principal design of a Power-Hardware-in-the-Loop setup 
1) Hardware: Damping the hardware reaction times can enhance the stability of the general system significantly. This damping for instance is achievable by adding inductive components between the power interface and the DUT or by slowing down the reaction time of active DUTs, though resulting in an affected DUT behavior.

2) Software: Software interfaces, usually referred to as interface algorithms, offer the opportunity of being adjusted to the individual system setup and thereby have a decent stability and accuracy. In-depth knowledge about the DUT and system behavior is a mandatory requirement for such an adjustment. Aiming at a wide range of DUTs without the necessity of modeling adapted interface algorithms, the Ideal Transformer Method (ITM) is implemented on most test facility setups as closed-loop stability can be achieved easily by damping the system (see Section III). Here, a bus voltage is used as the output of the RTS; the digital feedback loop is realized by an ideal current source fed by the low-pass filtered real currents (see Fig. 2). Stability analysis according to the Nyquist criterion shows the stabilizing effect of filter utilization [1].

Here again, while being a relatively stable interface algorithm with a good accuracy, stability does not come without a compromise - a low-pass filtering of the feedback currents improves the stability and independence from the DUT, but lowers the bandwidth and accuracy of the PHIL setup.

\section{Detailed Overview of Related Work}

PHIL in power grid applications is still a growing field of research and there are already several institutions, mostly universities and other research centers operating PHIL systems for research on power systems and their components. A classification of experimental setups described is proposed and more precisely portrayed in the following paragraphs.

\section{A. Co-Simulation Setups}

Co-simulation setups are co-interfaces of a not necessarily real-time capable simulation that may be combined with a PHIL approach by adding a power interface and hardware DUTs to the co-simulation.

Buescher etal., 2014 \& 2015, University of Oldenburg, Germany, describe the integration of a real photovoltaic system into the co-simulation framework mosaik. Mosaik is coupled with the commercial RTS by RTDS Technologies Inc. and both simulators are simulating a part of a low voltage grid with 52 nodes. Historical smart meter time-series data is used for the

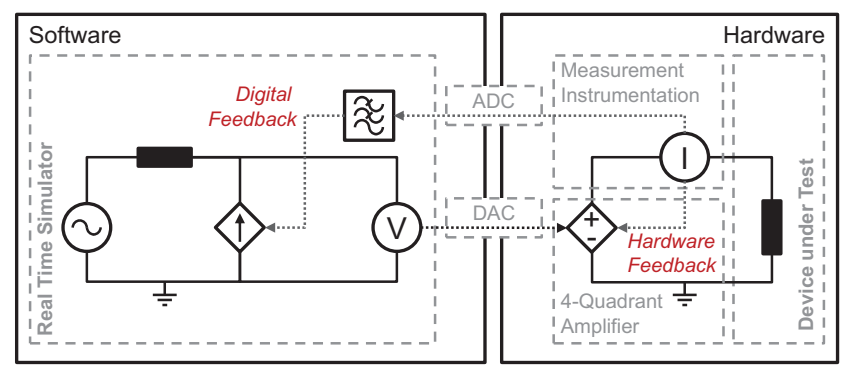

Fig. 2. Circuit diagram of a basic PHIL setup. simulation of the connected households and the distributed generation is simulated according to live measurements of a photovoltaic system. The approach focuses on long-term evaluations comprising large simulations without considering smart grid technologies. [2], [3]

Kochanneck etal., 2018, Karlsruhe Institute of Technology (KIT), Germany, are coupling a smart residential building (see Section V-A) with a co-simulation of a suburban low voltage distribution grid comprising intelligent buildings. The real building is connected to an artificial mains and a PVsimulator to emulate the most important electrical influences to the building. Soft- and hardware components are coupled via asynchronous calls utilizing a message bus for simplicity and robustness. [4]

Palmintier etal., 2015, National Renewable Energy Laboratory (NREL), USA, are evaluating the capabilities of PV inverters to provide system services. The inverters are connected to an artificial mains and a DC-supply for the simulation of the PV-arrays. The setpoints of both actors are derived in a multi-agent simulation of a distribution system and a weather simulation. The tested inverters are acting autonomously without exchanging control information with the simulator. [5]

\section{B. Static PHIL Setups}

Mather etal., 2013, National Renewable Energy Laboratory (NREL), USA, use a $500 \mathrm{~kW} / 200 \mathrm{~V}_{\mathrm{AC}} \mathrm{PV}$ inverter as DUT, connected to variable voltage sources on DC and $\mathrm{AC}$ side. Their experiments aim at proving different control modes of a PV inverter (constant $\cos (\phi)$, constant VAr) in a PHIL environment. No further information on feedback algorithms was given and it is not made clear whether an open-loop (more likely) or closed-loop was applied. However, if the system was run in closed-loop mode, figures and plots show that supposedly the ITM was used with a rather narrow bandwidth. This is because of the current-only feedback for the AC side and PV inverter behavior results plotted as power against $1000 \mathrm{~s}$. [6]

Langston et al., 2012, Florida State University, USA, describe the PHIL testing of a $500 \mathrm{~kW}$ photovoltaic (PV) inverter. They operate their system in open-loop mode for "inverter transient testing", where they ramp "the voltage magnitude and phase angle for individual phases as well as [...] the frequency of the references" [7]. No further information on temporal resolutions is given. For reactive power contributions of inverters, closed-loop testing is implemented. For the latter, a PI-controller with a time constant of approximately $50 \mathrm{~ms}$ for reference voltage tracking as well as a current feedback with a limited bandwidth to $60 \mathrm{~Hz}$ are used, which limits their system to static closed-loop investigations. [7]

Lauss et al., 2012, Austrian Institute of Technology (AIT), Austria, compare numerical simulations and hardware experiments with PHIL simulations. Their DUTs are 2 PV inverters connected to different nodes of a 3 node grid, which provide their standard $\mathrm{Q}(\mathrm{U})$ voltage control schemes. The results show slight differences in the transient time ranges, caused by the simulation time step of $50 \mu \mathrm{s}$, the bandwidth 
of the implemented ITM feedback method $(1 \mathrm{kHz})$, and their corresponding limited temporal resolution. Tests carried out at $10 \mu \mathrm{s}$ and $2 \mathrm{kHz}$ had an increased dynamic reaction, "but stability could not be achieved at all times". [8]

Seitl et al., 2014, Austrian Institute of Technology (AIT), Austria, implemented a battery model in a PHIL setup for system verification [9]. The system itself correspondingly represents the battery while the DUT represents the battery driven load. Temporal resolution of the utilized power amplifier with its PT2-behavior, a time constant of $1.5 \mu \mathrm{s}$, and the not closer described filtering of the ITMs feedback currents stabilize the system, so that rather long-term experiments are performed. The authors conclude with a maximum error below $2 \%$ and find their accuracy reasonably high for the field of research.

Kotsampopoulos et al., 2012, National Technical University of Athens, Greece, introduce a PHIL testbed driving a PV system. Theoretical considerations on feedback algorithms are made before their own system realization is explained in more detail. While not giving further information on the current feedback filter implemented, they describe that an ITM interface algorithm was used for the experiments in the time range of several hundred seconds. Stability issues are discussed, especially when introducing a gain in the feedback path in order to simulate a stronger DUT with equivalent behavior as the utilized DUT. [10].

Xi etal., 2015, Tsinghua University, China, use a PHIL experiment for analysis of voltage and frequency ancillary control of a wind energy conversion system (WECS) representing a voltage source. The focus lies on resulting small-signal stability of power systems and the comparison of software and PHIL simulations. A grid with four synchronous generators is emulated and represented by a $50 \mathrm{kVA} 4$-quadrant amplifier with a $3 \mathrm{~kW}$ WECS as DUT. For their closed-loop testing, the authors describe a low-pass filtering of the feedback current of utilized ITM and thus a decrease in accuracy. No additional information is given on the filter; but with the time scale of experiments in the range of several seconds and focus on power system oscillations around $0.7 \mathrm{~Hz}$ a rather low cutoff frequency can be assumed. [11]

Serban etal., 2017, Transilvania University of Brasov, Romania, present dynamic tests of a PV inverter as DUT and an induction motor and a resistive load in hardware are used to cause voltage and frequency events [12]. Microgrid behavior is emulated by a reduced-order frequency response model and thus not capable of interacting with higher frequency dynamics. The authors describe the voltage and current as feedback values, which, accumulated as power, are processed by the microgrid model. PHIL stability issues are not discussed any further. However, with the usage of the response model and space vectors instead of instantaneous values, stability is easier to achieve, leading to higher dynamics.

Karapanos etal., 2011, Delft University of Technology, Netherlands, describe their system setup and PHIL experiments with a Virtual Synchronous Generator (VSG). While they aim at providing virtual inertia and at testing the interaction between the VSG and the power interface driven by an RTDS simulator, they implemented physical filters at the power interface as well as at the VSG connection point. Those LCL filters have a relatively low cutoff frequency of $158 \mathrm{~Hz}$ which stabilizes their system. Total PHIL loop time is stated to be $0.85 \mathrm{~ms}$, leading all in all to a more static experimental scenario. [13]

\section{Dynamic PHIL Setups}

Lundstrom etal., 2013, National Renewable Energy Laboratory (NREL), USA, employed their PHIL system with unintenional islanding testing of two different PV inverters [14]. The test results were compared to those of hardware only experiments and show promising accuracy. However, closedloop feedback had a limited resolution, induced by the utilized amplifier (slew rate of $1 \mathrm{~V} / \mathrm{s}$, load change time of $300 \mu \mathrm{s}$ ) and a $1.2 \mathrm{kHz}$ cutoff frequency of the low-pass filtering applied to the feedback currents (ITM). The cutoff frequency was determined experimentally, with focus on system stability. [15]

\section{Transient PHIL Setups}

Schacherer etal., 2009, Karlsruhe Institute of Technology (KIT), Germany, present their results of a PHIL study testing a superconducting current limiter. [16]. As the DUT has a lower impedance than the emulated grid, they used the ITM with a voltage feedback for their loop, running with a simulation clock rate of $25 \mu \mathrm{s}$. To counter stability issues, which depend on impedance ratio and time delays, they limited their minimum source impedance to $0.95 \Omega$ with a $\mathrm{X} / \mathrm{R}$ ratio of 10. However, no further information on DUT impedance is given; the authors remark though, that their test setup stability limit could be found at a simulated system impedance of $0.69 \Omega$. Due to the restrictions of the experimental setup and the predictable behavior, Schacherer et al. were able to perform highly dynamic tests, i. e. to obtain a high resolution in short experiments.

\section{E. Theoretical Work on PHIL Testing}

In [17], Kotsampopoulos et al. consider advanced functions of PV inverters, such as $\mathrm{Q}(\mathrm{U})$ voltage control, $\mathrm{P}(\mathrm{f})$ droop and inertia control, and propose test procedures for ancillary services provided by those. Their approaches distinguish openloop and closed-loop scenarios, but without having a more detailed view on time scales. As a basic interface algorithm they suggest the ITM, where it is noted that "compensation measures may be needed to ensure stability or/and improve accuracy of the PHIL test" [17].

For detailed investigations on interface algorithms, a multitude of publications can be found-some of them mentioned in the following without going closer into detail: Lauss et al. [1] have a closer look at ITM feedback current filtering. In [18], Craciun et al. compare the ITM and damping impedance method for an PV integration. This method is modified by Paran et al. by using a variable damping impedance [19]. A multi-rating interface is proposed by Lehfuss et al. [20]. In [21], a more detailed and complex interfacing approach is proposed together with a method of rescaling power and voltage levels. 
TABLE I

Temporal and System Setup Classification of Published PHIL RESEARCH WORK

\begin{tabular}{lll}
\hline feedback & open-loop & closed-loop \\
\hline static & {$[6]$} & {$[6]^{1},[7],[8],[9],[10],[11],[12]$,} \\
& & {$[13]$} \\
dynamic & - & {$[14]$} \\
transient & {$[7]^{1}$} & {$[16]^{2}$} \\
\hline \hline
\end{tabular}

theoretical work [17], [1], [18], [19], [20], [21]

${ }^{1}$ possibly; no detailed information available

${ }^{2}$ restricted source and DUT impedance; passive DUT

\section{F. PHIL outside Power Grid Applications}

While this paper focuses on PHIL in power grid applications, there are also PHIL machine test beds and other applications that will not be further discussed at this point.

\section{G. Subsumption}

Most published test scenarios are focusing on rather static tests of renewable energy resources and their capability of providing ancillary services according to grid connection standards as well as beyond those, so called advanced functions. Only few publications report successful dynamic or transient PHIL testing. This is because of the principal necessity to test standard device behavior according to legislation as well as for testing the operability of the PHIL system itself, whereby the latter is a complex topic in need of further research. Energy system behavior and reaction, on the contrary, is seldom considered-what is likely explained by the rather small-scale test facilities and the rather small impact of the DUTs on the stability of real to-be-simulated interconnected grids.

Table I gives an overview of PHIL test beds and cosimulation setups. The classification is orientated at the content given in Table II.

\section{Grid Challenges And PHIL ReQuirements}

Main criteria for PHIL scenarios are the temporal resolution as well as the necessity of a closed-loop feedback. Modeling efforts are minimized and stability issues of the feedback mechanism are impeded by an adequate simulation setup, which can be advantageous especially for basic function tests of a wide range of DUTs. In contrast, for more specialized applications, the efforts of stabilizing the closed-loop system can be channeled specifically.

In the following, a classification and specification concept for power grid applications is proposed which clarifies the requirements on PHIL setups subject to temporal resolution and tested functionality or ancillary system services.

\section{A. Temporal Resolution}

In energy production, transmission, and grid regulation, a multitude of power quality issues is defined in standards, e.g. the European standard EN 50160 [22]. Based on the differentiation for the type and the duration of issues, different temporal requirements on the test system can be derived.
Unlike dynamic events, which require a high temporal resolution, quasi-static grid operation (e.g. unsymmetries and voltage deviations) can be simulated based on seconds or minutes. In contrast, transient events require a subsecond consideration of instantaneous values, owed to their short duration, e.g. lightning (LI) or switching impulses (SI). Obviously, transitions of areas are fluent, so that the following discussion is a basic concept:

1) Co-Simulation: As described in Section III-A, cosimulation is an approach for the simulation of heterogeneous systems and thus operating in similar temporal areas as static PHIL systems. When including closed-loop feedback, rather large cutbacks in accuracy must be made, especially due to rather low sample rates.

2) Static: For grid incidents taking place or only being relevant on a long-term time scale, e.g. violations of grid voltage, grid frequency and unsymmetries in LV grids, periods of seconds to minutes are of interest [22]. As a result, the behavior of connected grid components is only relevant in similar time scales. More specifically, experiments focus on DUT and grid behavior related to apparent power feed-in and thus on rms values of currents, instead of instantaneous ones. High resolution measurements however can be taken and evaluated independently with little effort.

3) Dynamic: In terms of lower harmonics, mains signaling, and more abstractly represented grid faults, higher dynamics in energy grids and DUT behavior are targeted and thus requested of PHIL test beds. Voltage fault durations, as defined in [22], are classified starting at $10 \mathrm{~ms}$. The frequency range of harmonic events covers the fault time range and is not closer defined. However, the standard considers harmonics up to the 25th in detail. This leads to a dynamic range of about $1 \mathrm{~Hz}$ to $1250 \mathrm{~Hz}$, where the latter value is equal to the 25 th harmonic in the European $50 \mathrm{~Hz}$ grid. Especially for realistic fault ride through and control algorithm stability testing scenarios, rather high PHIL test bed dynamics should be achieved.

4) Transient: The fastest and probably most complex case of PHIL studies is the detailed emulation of transients and specifically the closed-loop realization of active fault clearance and transient stability assessment. Those processes take place in the range of several $\mathrm{ms}$, e.g. SI $250 / 2500 \mu \mathrm{s}$, to less than $1 \mu \mathrm{s}$, e.g. LI $1.2 / 50 \mu \mathrm{s}$ [22]. As Section III shows, further research has to be carried out in order to achieve PHIL test beds capable of covering transient events as far as possible.

\section{B. Feedback Mechanism}

For the challenges arising from closed-loop PHIL systems, the necessity of feedback signals and grid reaction, e. g. for component testing, has to be considered. Especially for testing functions of simple active grid participants, e.g. photovoltaic or battery inverters, closing the loop might not be necessary and thus includes the risk of instability without having any benefit.

The closed-loop feedback mechanism, in contrary to passive component behavior testing, has its relevance especially in active ancillary service provisioning of grid connected devices 
as well as in the simulation of weak grid setups-whereas the impact of feedback currents on the artificial mains voltage is negligible in strong grid setups. Such strong grid setups, i.e. scenarios covering highly meshed or interconnected grids and comparably small loads or actors, will show little to none reaction and thus can be investigated in open-loop PHIL scenarios.

As for the reaction and behavior of grid components, standards like the German LV application rule VDE-AR-N 4105 [23] define the regulatory framework. While those standards only apply for certain power quality issues so far, a multitude of other grid services, i. e. advanced functions, are made possible with new connection technologies and grid requirements-which also need to be considered for PHIL testing and system classification:

1) Open-Loop: Open-loop PHIL studies-what basically leads to an incorrect wording-are here considered to be such experiments, where the DUT barely has an effect on the grid or the grid reaction is of minor interest. They are proposed in order to keep the whole setup as simple as possible, which, for stability reasons, is mandatory for emulation of transient grid events. Testing of standard DUT behavior for frequency stabilization, i. e. active power reduction $\mathrm{P}(\mathrm{f})$, is in focus of open-loop studies. However, experiments on communication, i. e. mains signaling, and fault behavior, e.g. fault ride through or conducted disturbances, are also applicable.

2) Closed-Loop: Starting from open-loop studies, for investigations of interdependencies between electrical components or energy grids and DUTs, closing the loop (see Fig. 2, digital feedback) in PHIL setups is fundamental. Specifically, testing of control algorithm stability and co-existance of a multitude of active systems especially in grids that can be considered to be rather weak-not last because of missing inertia-is of a high interest. While this is mostly relevant for islanded microgrids, also evening-out of unsymmetrical grid parts as well as the recognition and handling of grid faults and transients requires direct feedback of the DUT to the grid.

Above system setups of feedback mechanisms only consider simulated system behavior of arbitrarily complex grids or components. However, practical experiments in the PHIL test bed at KIT brought the insight that an additional abstraction layer in feedback behavior might be applicable, which allows for highly dynamic testing. This layer is a direct and accelerated hardware feedback by implementing an impedance simulation in the control loop of the power amplifier (see Fig. 2), in this case provided by advanced features from the utilized amplifier by Spitzenberger \& Spies $G m b H \& C o$. KG. The setup at KIT enhances a static open-loop PHIL simulation with such a hardware feedback, enabling to include a substitution impedance via internal amplifier settings. It should be mentioned that such a setup obviously leads to losses in accuracy and has a limited variability as well as range, but still promises comparably good results and will be investigated further.

A short summary of proposed PHIL requirements for different power quality issues and system services is given in Table II.
TABLE II

OVERView of PHIL-APPliCATIONS Related to POWER QuAlity AND Grid SERVICE Issues on DifFERENT TIME SCAlES

\begin{tabular}{|c|c|c|}
\hline feedback & open-loop & closed-loop \\
\hline grid & interconnection & islanded microgrid \\
\hline mains & strong, high inertia & weak, low inertia \\
\hline $\begin{array}{l}\text { co- } \\
\text { simulation }\end{array}$ & $\begin{array}{l}\text { secondary \& } \\
\text { tertiary reserve }(P(f))\end{array}$ & voltage support \\
\hline \multirow[t]{2}{*}{ static } & under-/overvoltages & unsymmetries \\
\hline & active power reduction $\mathrm{P}(\mathrm{f})$ & droop control, i. e. $\mathrm{Q}(\mathrm{U})$ \\
\hline \multirow[t]{2}{*}{ dynamic } & fault ride through & harmonics \\
\hline & mains signaling & control algorithm stability \\
\hline \multirow[t]{2}{*}{ transient } & transients, e.g. LI/SI & active fault clearance \\
\hline & conducted disturbances & transient stability assessment \\
\hline
\end{tabular}

\section{RESIDENTIAL PHIL ENVIRONMENT APPLICATION}

A PHIL environment is set up at the Karlsruhe Institute of Technology. It comprises the RTS and the associated power interface along with a smart residential building, inter alia, capable of providing ancillary system services for energy grids. In the course of ongoing research, different electric mains fault scenarios were designed and applied to the PHIL environment. A short introduction to the general setup and an exemplary test case are given in the following.

\section{A. Environment Setup}

The PHIL test bed utilizes an RTS of OPAL-RT TECHNOLOGIES, Inc. (1 core) and the grid simulation is done by the related software HYPERSIM. A PAS 30000 linear amplifier of Spitzenberger \& Spies interfaces the real time simulator as power interface to the DUT (rated power: 3x $10000 \mathrm{VA}$, lineto-line voltage: $400 \mathrm{~V}$, slew rate: $>52 \mathrm{~V} / \mu \mathrm{s}$ ).

The connected smart residential building, the KIT Energy Smart Home Lab (ESHL), is a laboratory environment for developing and testing smart grid hard- and software systems. It comprises distributed generation, flexible loads, and multiple storage technologies. The ESHL provides the area of application for the HESS that provides system services. A detailed description of the laboratory setup is given in [24].

Initially, the ESHL was built for the evaluation of the automated and multi-modal building energy management system described in [25] that optimizes the building's energy profile by scheduling the flexible entities according to user given goals. The communication system between the sensors, actors, and the management system is realized by a service-orientated middleware that allows for easy access to information and easy extension by new components, e.g. the power interface for hardware feedback [26].

\section{B. Application}

A grid fault scenario is applied in the previously described PHIL environment in order to test the HESS's advanced functionality - in this case, the provision of virtual inertia and primary reserve during a frequency drop. The frequency 


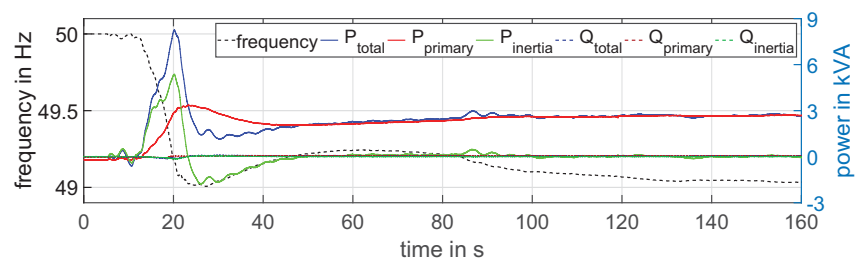

Fig. 3. Provision of virtual inertia and primary control by a HESS for the 2006 European blackout emulated in a PHIL test bed.

applied is the recorded frequency drop of the western European grid zone in 2006 after a mains synchronization loss. Simulation is carried out in open-loop as DUT behavior is in focus; anyhow, due to comparably low HESS power and a strong simulated LV grid, effects on the voltage level are negligible. Fig. 3 shows the frequency of the 3-phase $400 \mathrm{~V}$ grid provided as well as the HESS total active $(\mathrm{P})$ and reactive $(\mathrm{Q})$ power, separated by primary and inertia components As the figure and thus the PHIL experiment shows, the HESS as DUT is capable of supporting the grid in case of a frequency error by emulating the behavior of a synchronous generator's rotating mass.

\section{CONCLUSION}

This paper outlines the requirements for PHIL evaluations to emulate critical grid situations in LV grids. A classification approach is proposed and published literature on existing PHIL laboratories is analyzed and associated according to their qualities. Furthermore, an exemplary evaluation comprising a HESS in a PHIL environment at KIT is presented. Future work will implement closed-loop scenarios in the given test bed aiming at fastest-possible dynamic feedback realization under stable conditions.

\section{ACKNOWLEDGMENT}

We gratefully acknowledge the financial support from the German Federal Ministry for Economic Affairs and Energy (BMWi) for the projects Advanced Decentral Grid Control (funding no: 03ET7539F) and C/sells (funding no: 03SIN121) and from the Helmholtz Association for our participation in the research program Storage and Cross-linked Infrastructures.

\section{REFERENCES}

[1] G. Lauss, F. Lehfuss, A. Viehweider, and T. Strasser, "Power hardware in the loop simulation with feedback current filtering for electric systems," in IECON 2011. IEEE, 2011, pp. 3725-3730.

[2] M. Buescher, A. Claassen, M. Kube et al., "Integrated smart grid simulations for generic automation architectures with rt-lab and mosaik,' in IEEE Int. Conf. on Smart Grid Comm. IEEE, 2014, pp. 194-199.

[3] M. Buescher, K. Piech, S. Lehnhoff et al., "Towards smart grid system validation: Integrating the smartest and the sesa laboratories," in IEEE 24th Int. Symposium on Ind. Electronics (ISIE 2015). IEEE, 2015, pp. 1144-1150.

[4] S. Kochanneck, I. Mauser, K. Phipps, and H. Schmeck, "Hardware-inthe-loop co-simulation of a smart building in a low-voltage distribution grid," in accepted by: Innovative Smart Grid Technologies Conference Europe (ISGT-Europe), 2018 IEEE PES. IEEE, 2018, pp. 1-6.

[5] B. Palmintier, B. Lundstrom, S. Chakraborty et al., "A power hardwarein-the-loop platform with remote distribution circuit cosimulation," IEEE Trans. on Ind. Electronics, vol. 62, no. 4, pp. 2236-2245, 2015.
[6] B. A. Mather, M. A. Kromer, and L. Casey, "Advanced photovoltaic inverter functionality verification using $500 \mathrm{kw}$ power hardware-in-loop (phil) complete system laboratory testing," in IEEE PES Innovative Smart Grid Tech. Conf. (ISGT). IEEE, 2013, pp. 1-6.

[7] J. Langston, K. Schoder, M. Steurer et al., "Power hardware-in-the-loop testing of a $500 \mathrm{kw}$ photovoltaic array inverter," in IECON 2012. IEEE, 2012, pp. 4797-4802

[8] G. Lauss, F. Lehfuss, B. Bletterie et al., "Examination of lv grid phenomena by means of phil testing," in IECON 2012. IEEE, 2012, pp. 4771-4776.

[9] C. Seitl, J. Kathan, G. Lauss, and F. Lehfuss, "Power hardware-inthe-loop implementation and verification of a real time capable battery model," in 2014 IEEE 23rd Int. Symposium on Ind. Electronics (ISIE). IEEE, 2014, pp. 2285-2290.

[10] P. Kotsampopoulos, V. Kleftakis, G. Messinis, and N. Hatziargyriou, "Design, development and operation of a phil environment for distributed energy resources," in IECON 2012. IEEE, 2012, pp. 4765-4770.

[11] X. Xi, H. Geng, G. Yang et al., "Small-signal stability analysis and phil experiment validation of power system with wecs voltage/frequency ancillary control," in IECON 2015 - Yokohama, K. Ohishi and H. Hashimoto, Eds. IEEE, 2015, pp. 002 544-002549.

[12] I. Serban and C. P. Ion, "A phil system designed for testing the dynamic response of microgrid units," in IEEE Int. Conf. on Environment and Electrical Engineering and IEEE Ind. and Comm. Power Systems Europe (EEEIC/I \& CPS Europe). IEEE, 2017, pp. 1-6.

[13] V. Karapanos, S. de Haan, and K. Zwetsloot, "Real time simulation of a power system with vsg hardware in the loop," in IECON 2011. IEEE, 2011, pp. 3748-3754.

[14] B. Lundstrom, B. Mather, M. Shirazi, and M. Coddington, "Implementation and validation of advanced unintentional islanding testing using power hardware-in-the-loop (phil) simulation," in 2013 IEEE 39th Photovoltaic Specialists Conf. (PVSC). IEEE, 2013, pp. 3141-3146.

[15] B. R. Lundstrom, "An advanced platform for development and evaluation of grid interconnection systems using hardware-in-the-loop," Master Thesis, Colorado School of Mines, Golden, Colorado, 2013.

[16] C. Schacherer, J. Langston, M. Steurer, and M. Noe, "Power hardwarein-the-loop testing of a ybco coated conductor fault current limiting module," IEEE Trans. on App. Superconductivity, vol. 19, no. 3, pp. 1801-1805, 2009.

[17] P. Kotsampopoulos, N. Hatziargyriou, B. Bletterie, G. Lauss, and T. Strasser, "Introduction of advanced testing procedures including phil for dg providing ancillary services," in IECON 2013. IEEE, 2013, pp. 5398-5404.

[18] B.-I. Craciun, T. Kerekes, D. Sera, R. Teodorescu et al., "Grid integration of pv power based on phil testing using different interface algorithms," in IECON 2013. IEEE, 2013, pp. 5380-5385.

[19] S. Paran, F. Fleming, D. Li, and C. S. Edrington, "Utilization of adaptive phil interfaces for harmonic load cases," in IECON. IEEE, 2014, pp. 3803-3808.

[20] F. Lehfuss, G. Lauss, and T. Strasser, "Implementation of a multi-rating interface for power-hardware-in-the-loop simulations," in IECON 2012. IEEE, 2012, pp. 4777-4782.

[21] P. C. Kotsampopoulos, F. Lehfuss, G. F. Lauss et al., "The limitations of digital simulation and the advantages of phil testing in studying distributed generation provision of ancillary services," IEEE Trans. on Industrial Electronics, vol. 62, no. 9, pp. 5502-5515, 2015.

[22] DIN EN 50160:2011-02, "Voltage characteristics of electricity supplied by public distribution networks; German version EN 50160:2010 + Cor. :2010," DKE Deutsche Kommission Elektrotechnik Elektronik Informationstechnik im DIN und VDE, 2011.

[23] VDE-AR-N 4105:2011-08, “Generators connected to the low-voltage distribution network - Technical requirements for the connection to and parallel operation with low-voltage distribution networks," Forum Netztechnik/Netzbetrieb im VDE (FNN), 2011.

[24] S. Kochanneck, I. Mauser, B. Bohnet et al., "Establishing a hardwarein-the-loop research environment with a hybrid energy storage system," in IEEE Innovative Smart Grid Tech.-Asia. IEEE, 2016, pp. 497-503.

[25] I. Mauser, J. Müller, F. Allerding, and H. Schmeck, "Adaptive building energy management with multiple commodities and flexible evolutionary optimization," Renewable Energy, vol. 87, Part 2, pp. 911 - 921, 2016.

[26] K. Bao, I. Mauser, S. Kochanneck et al., "A microservice architecture for the intranet of things and energy in smart buildings: Research paper," in Proc. o. t. 1st Int. Workshop on Mashups of Things and APIs, ser. MOTA '16. ACM, 2016, pp. 3:1-3:6. 